\title{
Use of Frenum in Determining the Original Vertical Position of Teeth
}

\author{
Prasad $K D^{1}$, Alva $H^{2}$
}

\begin{abstract}
Purpose: To determine the functional position of labial, lingual and buccal frenii with the corresponding central incisor, premolar or molars in natural dentition.

Materials and method: 20 dentate subjects between 20-40years of age were selected for the study. Maxillary and mandibular impressions were made and the distance from anterior attachment of frenum to the cusp tip of the corresponding tooth was measured.

Result: The mean distance between the anterior attachment of labial frenum to the incisal edge of incisor was $12.25 \mathrm{~mm}$, the mean distance between the anterior attachment of buccal frenum of right side to cusp tip of corresponding teeth was $12.75 \mathrm{~mm}$ and left side was $12.8 \mathrm{~mm}$ and the mean distance between the anterior attachment of lingual frenum to incisal edge of incisor was $15.4 \mathrm{~mm}$. The coefficient of variation were $14.7 \%, 16.65 \%, 15.75 \%$ and $6.4 \%$ respectively.

Conclusion: The distance between the anterior attachment of the frenum to the cusp tips/incisal edges can be used as a pre-extraction record for determining the original vertical position of teeth.
\end{abstract}

Keywords: Anterior attachment, Vertical dimension at occlusion

${ }^{1} \mathrm{MDS}$

Professor

Department of Prosthodontics

AB Shetty Memorial Institute of Dental Sciences, Deralakatte, Mangalore, Karnataka

${ }^{2} P G$ student

Department of Prosthodontics

AB Shetty Memorial Institute Of Dental Sciences,

Deralakatte, Mangalore, Karnataka

\section{Contact Author \\ Dr. Harshitha Alva \\ drharshitha@gmail.com}

J Oral Health Comm Dent 2013;7(1)44-46

\section{INTRODUCTION}

V

ertical dimension refers to length of the face as determined by the amount of separation of the jaws.

Recording the vertical dimension is one of the most important steps in the fabrication of complete dentures. Many methods have been used to determine VDO like interocclusal records, swallowing, closest speaking space, cephalometric radiographs, certain anatomic landmarks and lip length (1).

But none of these methods have proven to be perfectly accurate. All estimates of vertical dimension cannot be considered totally accurate until the teeth are arranged on their trial bases, and phonetic and aesthetic aspects are checked against the relations established. Few authors have suggested the use of pre-extraction records for determining the VDO (2-4). Pre-extraction diagnostic casts facilitate determination of
VDO and complete denture fabrication.

The purpose of this study is to correlate the distance between the anterior attachment of labial, lingual and buccal frenii to the corresponding incisors and premolars or molars to be used as pre-extraction records.

\section{MATERIALS AND METHODS}

20 dentate subjects $(10$ men and 10 women) between 20-40years of age were selected for the study. Subjects with class I skeletal and dental relationships with no prosthetic replacement were included and subjects with class II or class III relationship, fractured or abraded teeth and ankyloglossia were excluded from the study (1).

Impression trays were selected such that at least 2-3mm space for the impression material is available and the tray covered the most distal tooth. Impression material was 
mixed according to the manufacturer's instructions. The patient was seated in an upright position. For accurately recording the frenum in maxilla, lips and cheeks are retracted and the loaded tray is inserted into the patients mouth first in the posterior and then anterior region. The lip is first elevated and then extended outwards, downwards and inward. Then the cheek is retracted, pulled outward, downward and inward.

For mandible, the labial frenum and flange is recorded by moving the lip in outward, upward and inward direction and buccal frenum was recorded by moving the cheek outward, upward and backward directions. The lingual frenum can be recorded by asking the patient to protrude his tongue out and then touch the anterior part of the palate (1). Maxillary and mandibular impression of each patient was made with irreversible hydrocolloid impression material (Zelgan plus) and casts were poured with dental stone.

A two pour technique was followed to build the casts. Now the distance between the anterior attachment of labial frenum to incisal edge of incisor, buccal frenii to molars or premolar in maxilla and the distance between anterior attachment of lingual frenum to incisal edge of incisors is measured using a divider and scale. The results were then calculated and statistically analysed.

\section{RESULTS}

The mean distance between the anterior attachment of labial frenum to the incisal edge of incisor in maxilla was $12.25 \mathrm{~mm}$, the mean distance between the anterior attachment of buccal frenum of right and left side to cusp tip of corresponding teeth

Table 1: measurements, means and standard deviation (SD) in millimetres

\begin{tabular}{|c|c|c|c|c|}
\hline \multirow[t]{2}{*}{ SUBJECTNO: } & \multicolumn{3}{|c|}{ MAXILLA } & \multirow{2}{*}{$\frac{\text { MANDIBLE }}{\text { LINGUAL }}$} \\
\hline & LABIAL & $B U C C A L(R)$ & $B U C C A L(L)$ & \\
\hline 1 & 10 & 15 & 16 & 17 \\
\hline 2 & 10 & 15 & 13 & 14 \\
\hline 3 & 11 & 13 & 12 & 17 \\
\hline 4 & 14 & 14 & 12 & 15 \\
\hline 5 & 13 & 13 & 14 & 15 \\
\hline 6 & 11 & 15 & 13 & 16 \\
\hline 7 & 10 & 14 & 14 & 16 \\
\hline 8 & 11 & 14 & 15 & 16 \\
\hline 9 & 12 & 15 & 14 & 16 \\
\hline 10 & 14 & 13 & 12 & 15 \\
\hline 11 & 13 & 12 & 13 & 15 \\
\hline 12 & 15 & 11 & 12 & 14 \\
\hline 13 & 14 & 10 & 11 & 15 \\
\hline 14 & 15 & 11 & 12 & 14 \\
\hline 15 & 11 & 10 & 10 & 15 \\
\hline 16 & 12 & 15 & 16 & 16 \\
\hline 17 & 15 & 9 & 10 & 16 \\
\hline 18 & 10 & 15 & 16 & 17 \\
\hline 19 & 11 & 9 & 9 & 15 \\
\hline 20 & 13 & 12 & 12 & 14 \\
\hline MEAN & 12.25 & 12.75 & 12.8 & 15.4 \\
\hline SD & 1.803 & 2.124 & 2.016 & 0.995 \\
\hline
\end{tabular}

The results of this study revealed that mean distance between the anterior attachment of labial frenum in maxilla to the incisal edge of the corresponding incisor was $12.25 \mathrm{~mm}$ with a standard deviation of 1.803 , the mean distance between the anterior attachment of buccal frenum of right side to corresponding tooth was 12.75 with a standard deviation of 2.124 , mean distance on left side being $12.8 \mathrm{~mm}$ with standard deviation of 2.016 and the distance between the anterior attachment of the lingual frenum to the incisal edge of the corresponding incisor was $15.4 \mathrm{~mm}$ with a standard deviation of 0.995 . Coefficient of variation was found to be least for lingual frenum indicating the distance to be most consistent.

The incisive papilla is stable and can be used for determining the VDO of edentulous patients. However this study gives the mean distance of the teeth from the frenum and states it to be reliable when the frenum is recorded in function. Because the incisal edges and cusp tips of the teeth are generally stable, the position of the frenum can be considered relatively stable when the frenum is recorded in function. 


\section{CONCLUSIONS}

Within the limitations of this study, the following conclusions were drawn:

1. There exist a correlation between the anterior attachment of the frenum and the corresponding teeth.

2. The distance between the anterior attachment of frenum and the incisal edge or cusp tip of corresponding teeth is reliable when recorded in function.

3. This distance can be used to determine the original vertical position of the teeth during complete denture fabrication.

\section{REFERENCES}

1. Bissasu M. Use of lingual frenum in determining the original vertical position of mandibular anterior teeth. $\mathrm{J}$ Prosthet dent 1999;82:177-181

2. Silverman MM. The speaking method in measuring vertical dimension. J Prosthet Dent 1953;3:193-199

3. Turner LC. The profile tracer: method for obtaining accurate pre-extraction records. J Prosthet Dent 1969;21:364370.

4. Smith DE. The reliability of pre-extraction records for complete dentures. J Prosthet Dent 1971;25:592-608. 